\title{
A Sensitivity Study of Conductivity Values in the Passive Bidomain Equation
}

\author{
Peter R. Johnston \\ School of Biomolecular and Physical Sciences, \\ and \\ Queensland Micro- and Nanotechnology Centre, \\ Griffith University, Nathan, QLD, Australia, 4111
}

\begin{abstract}
There is a complex interplay between the four conductivity values used in the bidomain equation and the resulting electric potential distribution in cardiac tissue arising from subendocardial ischaemia. Based on the three commonly used experimentally derived conductivity data sets, a non-dimensional formulation of the passive bidomain equation is derived, which gives rise naturally to several dimensionless conductivity ratios. The data sets are then used to define a parameter space of these ratios, which is studied by considering the correlation coefficients between different epicardial potential distributions.

From this study, it is shown that the ratio of the intracellular longitudinal conductivity to the intracellular transverse conductivity is the key parameter in explaining the differences between the epicardial potential distributions observed with these three data sets.

Keywords: Bidomain equation, Electrocardiography, Mathematical Model, Dimensionless Formulation, Conductivity Values, Subendocardial Ischaemia
\end{abstract}

\section{Introduction}

The intracellular $(i)$ and extracellular $(e)$ longitudinal $(l)$ and transverse $(t)$ conductivities $g_{i l}, g_{e l}, g_{i t}$ and $g_{e t}$ combine in a complex fashion to produce 
electric potential distributions in cardiac tissue. Given the electrocardiographic importance of ST-segment depression and elevation as a diagnostic tool, several studies have been undertaken, which look at the effect these conductivity parameters have on epicardial potential distributions arising from subendocardial ischaemia $[1,2,3,4,5,6]$. Generally, the conductivity parameters used in these simulations have come from detailed experimental measurements of four conductivities $[7,8,9]$. However, due to large variability, and therefore high uncertainty, in these values, researchers have used simulation techniques in an attempt to derive consistent conductivity values $[10,11]$.

The initial simulation studies, of the effect of conductivity values on epicardial potential distributions arising from subendocardial ischaemia $[1,2$, 3, 4], merely pointed out that the different conductivity data sets yield significantly different epicardial potential distributions. A more detailed conductivity parameter study has been undertaken [6], using a parameter space based on variations in conductivity values obtained via simulation studies [11]. The main focus of the above parameter study was to observe current loops within tissue and explain the differences in epicardial potential distributions induced by varying the degree of subendocardial ischaemia.

The aim of the present study is to explain the differences, in epicardial potential distributions, which arise from the three experimentally measured conductivity data sets $[7,8,9]$. The model underlying this study is the passive bidomain equation which is rendered dimensionless in such a fashion that, for the three conductivity data sets, the left-hand side of the governing equation is approximately independent of the data chosen and all changes in epicardial potential distributions can be explained by studying the righthand side of the governing equation [12]. Analysis of the right-hand side 
shows that the ratios of conductivity values $g_{i l} / g_{i t}$ and $g_{e t} / g_{i t}$ arise naturally from this dimensionless formulation of the problem. The ratio $g_{b} / g_{i t}$, where $g_{b}$ is the conductivity of blood, also comes from the boundary conditions of the normalised formulation. These three ratios are different to those used in [6], but can be derived from the ratios studied therein.

The present study is undertaken by looking at ranges of the three abovementioned ratios which are derived from the three measured conductivity data sets. The resulting parameter space does not intersect with that considered in [6], which, as discussed above, was motivated by simulation studies [11].

The significance of the research undertaken here is that it provides an explanation of the effects that different experimentally measured sets of conductivity data have on the epicardial potential distribution. These conductivity sets, as mentioned above, have been shown to exhibit markedly different epicardial potential distributions, for a given geometry of cardiac tissue and degree of subendocardial ischaemia. Further, the study is restricted to these three data sets, as they all yield a value of the total longitudinal conductivity to total transverse conductivity ratio that is very close to 3.2 , which, as will be shown later, means that the left hand side of the governing differential equation is approximately independent of the conductivity data set chosen.

The remainder of the paper is set out as follows. In the next section the governing equations and boundary conditions are described and the nondimensional formulation is established. Also, the model geometry is described and the parameter space defined. Section 3 presents results of the simulations in terms of epicardial potential distributions, and these are compared via the correlation coefficient. In Section 4, the results of the simulations are discussed in the context of previous simulation studies and the conclusions 
from these results are related to previous biophysical observations. Finally, overall conclusions are drawn regarding the study.

\section{Methods}

\subsection{Governing Equations}

The passive bidomain equation governs the electric potential in a region of cardiac tissue during the ST segment of the electrocardiogram and is given by $[6]$

$$
\nabla \cdot\left(\mathbf{M}_{i}+\mathbf{M}_{e}\right) \nabla \phi_{e}=-\nabla \cdot \mathbf{M}_{i} \nabla \phi_{m}
$$

where $\phi_{e}$ is the extracellular potential in the cardiac tissue, $\phi_{m}$ is the specified transmembrane potential distribution and $\mathbf{M}_{i}$ and $\mathbf{M}_{e}$ represent conductivity tensors for the intracellular $(i)$ and extracellular $(e)$ spaces, respectively. These conductivity tensors contain information about the local fibre direction.

The conductivity tensors take the form [13]

$$
\mathbf{M}_{h}=\mathbf{A G}_{h} \mathbf{A}^{T}
$$

for $h=i$ or $e$, where $\mathbf{G}_{h}$ is a diagonal matrix containing the conductivity values along the fibre direction, $g_{h l}$, across the sheets of fibres, $g_{h t}$, and between the sheets of fibres, $g_{h n}$. Here it will be assumed that $g_{h t}=g_{h n}$. The matrix $\mathbf{A}=\left\{a_{i j}\right\}$ is a rotation matrix mapping the local fibre direction into the global coordinate system. Under these assumptions it can be shown that

$$
\mathbf{M}_{h}=g_{h t} \mathbf{I}+\left(g_{h l}-g_{h t}\right) \hat{\mathbf{a}}^{T} \hat{\mathbf{a}}
$$

where $\mathbf{I}$ is the $3 \times 3$ identity matrix and $\hat{\mathbf{a}}$ is the unit vector indicating the local direction of the fibres [14]. 
It will be further assumed that the cardiac tissue is in contact with a blood mass. The electric potential in the blood mass, $\phi_{b}$, is governed by Laplace's equation

$$
\nabla^{2} \phi_{b}=0
$$

\subsection{Model Geometry}

For the purposes of this study it will be assumed that the computational domain is represented by a slab of cardiac tissue, finite in the $x$ and $y$ directions and of unit thickness in the $z$-direction. The origin of the rectangular $(x, y, z)$ coordinate system is placed in the centre of the tissue slab with the epicardial surface represented by the $x-y$ plane $(z=0)$ and, since the cardiac tissue is of unit thickness, the endocardial surface is at $z=1$. To mimic contact of the cardiac tissue with a blood in a ventricle, the endocardial surface is assumed to be in contact with a blood mass of finite size in the $z$-direction.

Placed at the centre of the slab of tissue is a region of partial thickness ischaemia, extending from the endocardium towards the epicardium. This region of ischaemia is parallelepipedal in shape, with sides aligned with the $x$ and $y$ coordinate axes, as shown in Figure 1.

The transition, between the normal and ischaemic tissue, is described by a smooth variation in the transmembrane potential during the ST segment. The difference in plateau transmembrane potential between normal and ischaemic tissue, $\Delta \phi_{p}$, is set at $-30 \mathrm{mV}$ and the transition is described in terms of exponential and hyperbolic functions $[15,1]$.

For this study, it will be assumed that fibres rotate through an angle of $120^{\circ}$ (chosen for consistency with previous models $[1,3]$ ), moving from the epicardium to the endocardium (as shown in Figure 1). The fibres on the epicardium are aligned along the positive $x$-axis, and so the longitudinal 
direction at any depth $z$, with respect to the positive $x$-axis, is given by

$$
g(z)=\frac{2 \pi}{3} z,
$$

with the fibres on the endocardium rotated through $120^{\circ}$. As mentioned above, this rotation is taken into account in the conductivity tensors by assuming that

$$
\mathbf{A}=\left(\begin{array}{ccc}
\cos g(z) & \sin g(z) & 0 \\
-\sin g(z) & \cos g(z) & 0 \\
0 & 0 & 1
\end{array}\right)
$$

\subsection{Boundary Conditions}

To solve the governing equations, several boundary conditions are required. It will be assumed that the epicardial surface is insulated, i.e.

$$
\text { at } z=0, \quad \frac{\partial \phi_{e}}{\partial z}=0
$$

which is chosen for consistency with previous simulations [1,3] and to match experimental protocols, where epicardial potentials were measured in an open chest experiment [16]. It will also be assumed that the edges of the tissue and the blood mass in the $x$ and $y$ directions are insulated, i.e.

$$
\begin{array}{cl}
\mathbf{M}_{e} \nabla \phi_{e} \cdot \mathbf{n}=0 & \text { for } \quad 0 \leq z \leq 1 \\
\nabla \phi_{b} \cdot \mathbf{n}=0 & \text { for } \quad z \geq 1
\end{array}
$$

where $\mathbf{n}$ is the appropriate outward pointing normal. At the bottom of the blood mass, the potential is set to zero. Finally, continuity of potential and current is assumed between the tissue and the blood mass, so at $z=1$

$$
\phi_{e}=\phi_{b} \quad \text { and } \quad g_{e t} \frac{\partial \phi_{e}}{\partial z}=g_{b} \frac{\partial \phi_{b}}{\partial z}
$$

where $g_{b}$ is the conductivity of blood. These boundary conditions are chosen

for consistency with previous studies $[1,3,4]$, in which it was argued that, 
given the scale of the problem under consideration, these boundary conditions are justified [17]. The boundary conditions have been reconsidered recently and modified [18], but the modifications have been shown to make only a small difference when modelling ST segment shifts [19].

\subsection{Non-dimensional Formulation}

To facilitate easier study, the governing equations will be rendered nondimensional by normalising the potentials, relative to the difference between normal and ischaemic transmembrane potentials, $\Delta \phi_{p}$, and normalising the conductivity tensors with respect to the quantity $g_{i t}+g_{e t}$. Define the dimensionless potentials $\Phi_{m}=\phi_{m} / \Delta \phi_{p}, \Phi_{e}=\phi_{e} / \Delta \phi_{p}$ and $\Phi_{b}=\phi_{b} / \Delta \phi_{p}$ and the dimensionless tensors

$$
\mathbf{N}_{i}=\frac{\mathbf{M}_{i}}{g_{i t}+g_{e t}} \quad \text { and } \quad \mathbf{N}_{e}=\frac{\mathbf{M}_{e}}{g_{i t}+g_{e t}}
$$

Using this notation the governing equations (1) and (4) become

$$
\nabla \cdot\left(\mathbf{N}_{i}+\mathbf{N}_{e}\right) \nabla \Phi_{e}=-\nabla \cdot \mathbf{N}_{i} \nabla \Phi_{m}
$$

and

$$
\nabla^{2} \Phi_{b}=0
$$

with appropriate expressions for the boundary conditions. The tissue/blood interface conditions are now that

$$
\Phi_{e}=\Phi_{b} \quad \text { and } \quad \frac{g_{e t}}{g_{i t}+g_{e t}} \frac{\partial \Phi_{e}}{\partial z}=\frac{g_{b}}{g_{i t}+g_{e t}} \frac{\partial \Phi_{b}}{\partial z}
$$

Using the notation of Potse et al. [14], the conductivity tensor $\mathbf{N}_{i}+\mathbf{N}_{e}$ can be rewritten as

$$
\mathbf{N}_{i}+\mathbf{N}_{e}=\frac{\mathbf{M}_{i}+\mathbf{M}_{e}}{g_{i t}+g_{e t}}=\mathbf{I}+(\delta-1) \mathbf{a}^{T} \mathbf{a}
$$


where

$$
\delta=\frac{g_{i l}+g_{e l}}{g_{i t}+g_{e t}}
$$

and $\mathbf{a}$ is a unit vector describing the fibre direction. The quantity $\delta$ is the ratio of the total longitudinal conductivity to the total transverse conductivity. As shown previously [12], to a good approximation, $\delta$ takes the value 3.2 for each of the three conductivity data sets of interest. Hence, to a good approximation, the left hand side of equation (12) is independent of the conductivity data set chosen. A consequence of this is that all differences between conductivity data sets come from the right hand side of equation (12). For the remainder of this paper $\delta$ will be fixed at the value of 3.2.

\subsection{Conductivity Ratios}

The conductivity tensor $\mathbf{N}_{i}$ takes the form

$$
\mathbf{N}_{i}=\frac{g_{i t}}{g_{i t}+g_{e t}} \mathbf{I}+\frac{g_{i l}-g_{i t}}{g_{i t}+g_{e t}} \mathbf{a}^{T} \mathbf{a}
$$

which contains two dimensionless groups

$$
\frac{g_{i t}}{g_{i t}+g_{e t}}=\frac{1}{1+\frac{g_{e t}}{g_{i t}}}
$$

and

$$
\frac{g_{i l}-g_{i t}}{g_{i t}+g_{e t}}=\frac{\frac{g_{i l}}{g_{i t}}-1}{1+\frac{g_{e t}}{g_{i t}}} .
$$

As noted previously [12], there are two conductivity ratios which occur naturally in this dimensionless formulation, namely

$$
\frac{g_{i l}}{g_{i t}} \text { and } \frac{g_{e t}}{g_{i t}}
$$

A third conductivity ratio also comes from the right hand side of the continuity of current boundary condition (equation (14)), i.e.

$$
\frac{g_{b}}{g_{i t}+g_{e t}}=\frac{\frac{g_{b}}{g_{i t}}}{1+\frac{g_{e t}}{g_{i t}}} .
$$


The ratios $g_{i l} / g_{i t}, g_{e t} / g_{i t}$ and $g_{b} / g_{i t}$ are summarised for the three experimentally determined conductivity data sets in Table 1 . The value of $g_{b}$ is taken to be the generally accepted value of $0.67 \mathrm{~S} / \mathrm{m}$ [20]. It should also be noted that the quantity $g_{e l}$ does not appear explicitly in this non-dimensional formulation.

\subsection{Study Parameters}

Given the range of conductivity values displayed in Table 1, the parameter space for the sensitivity study is created as follows:

1. Let $g_{i l} / g_{i t}$ take the values $5,7,9,11$.

2. Let $g_{e t} / g_{i t}$ take the values $1,5,9,13$.

3. Let $g_{b} / g_{i t}$ take on the values $10,15,25,35$.

4. Calculate the value of $g_{e l} / g_{i t}$ from the fact that $\delta=3.2$, i.e.

$$
\frac{g_{e l}}{g_{i t}}=\delta\left(1+\frac{g_{e t}}{g_{i t}}\right)-\frac{g_{i l}}{g_{i t}} .
$$

There are two things to observe from this parameter space. Firstly, the data of Roberts and Scher [9] sit very close to one corner of the parameter space, whereas the other two sets are quite close to the centres of faces of the three dimensional parameter space. Secondly, when $g_{e t} / g_{i t}=1$, all values of $g_{i l} / g_{i t}>5$ lead to negative values of $g_{e l} / g_{i t}$, which is physiologically unrealistic. These points were removed from the study, which further isolates the data of Roberts and Scher.

Equation (16) exposes some interesting relationships between the conductivity values. Firstly, increasing the intracellular longitudinal conductivity, $g_{i l}$, effectively reduces the extracellular longitudinal conductivity. On the other hand, increasing the extracellular transverse conductivity, $g_{e t}$, effectively increases the extracellular longitudinal conductivity, thus increasing the overall extracellular conductivity. 
The parameter space defined here is in some aspects different to that chosen by Hopenfeld et al. [6]. Using the parameters defined for this study, the parameter space in [6] was given by $1 \leq g_{e t} / g_{i t} \leq 250,13<g_{b} / g_{i t}<1000$ and $20 \leq g_{i l} / g_{i t} \leq 50$. Whilst the ranges of the parameters $g_{e t} / g_{i t}$ and $g_{b} / g_{i t}$ considered here are a subset of those considered by Hopenfeld et al., the ranges for the parameter $g_{i l} / g_{i t}$ do not overlap at all. The range chosen in [6] was based on a previous simulation study of modelled cardiac tissue, whereas the range chosen here is based on experimental measurements.

To compare the effect of the different conductivity values, the dimensionless governing equations (12) and (13) were solved, subject to the given boundary conditions, using the finite volume method [21]. The point of comparison is a visual inspection of the epicardial surface potentials, along with measures of the correlation coefficient given by

$$
C C=\frac{\sum_{i=1}^{n}\left[\left(\phi_{1}\right)_{i}-\overline{\phi_{1}}\right]\left[\left(\phi_{2}\right)_{i}-\overline{\phi_{2}}\right]}{\left\|\phi_{1}-\overline{\phi_{1}}\right\|\left\|\phi_{2}-\overline{\phi_{2}}\right\|}
$$

where $\phi_{1}$ and $\phi_{2}$ are vectors representing two epicardial potential distributions and $\overline{\phi_{j}}$ is the average value of the distribution $\phi_{j}, j=1,2$. The correlation coefficient is chosen as a means of comparing two potential distributions as it is routinely used in electrocardiography to compare both body surface and heart surface potential distributions [22].

\section{Results}

For this study, a slab of cardiac tissue of size $16 \mathrm{~cm} \times 16 \mathrm{~cm}$ in the $x$ and $y$ directions and of thickness $1 \mathrm{~cm}$, as described previously [1], was used. The governing equations were solved using the finite volume method [21], in which the blood mass had a depth of $26 \mathrm{~cm}$, the bottom face of which was set as the zero of potential. The centrally located ischaemic region had a size of 
$4 \mathrm{~cm} \times 4 \mathrm{~cm}$ in the $x$ and $y$ directions and covered $50 \%$ of the depth of the tissue. All ischaemic borders were set at a value of 0.01 , leading to a sharp interface between normal and ischaemic tissue. Finally, as mentioned above, fibre rotation was set at $120^{\circ}$ [23].

Figure 2 shows the epicardial potential distributions, obtained by solving the non-dimensional governing equations (12) and (13) for the conductivity data sets of (a) Clerc [7], (b) Roberts et al. [8] and (c) Roberts and Scher [9]. The conductivity data sets of Clerc and Roberts et al. show three peaks of potential, yet the data of Roberts and Scher show only one peak. Also, the potential distributions (a) and (b) in Figure 2 show large potential gradients above the ischaemic boundary, which is a feature of experimental observations [16], whereas Figure 2(c) does not show such gradients. The object of this study is to attempt to explain these differences.

Figure 3 shows a combined set of epicardial potential distributions with the ratio $g_{b} / g_{i t}$ set to 10 . The ratio $g_{e t} / g_{i t}$ varies across the rows and the ratio $g_{i l} / g_{i t}$ varies down the columns. Note that the panel at the top left (as indicated by the ${ }^{*}$ ) corresponds approximately to the data of Roberts and Scher [9]. Note also that there are no figures in rows 2, 3 and 4 of column 1 due to the fact that these combinations of parameters yield negative values for the conductivity ratio $g_{e l} / g_{i t}$, as discussed previously. The pair of numbers under each figure represents correlation coefficients (equation (23)). The first number in each pair is the correlation coefficient across the rows of the figure, where the values are determined relative to the distribution in that row with $g_{e t} / g_{i t}=5$ (column 2). The second number of the pair is the correlation coefficient down the columns of the figure, where the values are determined relative to the distribution in that column with $g_{i l} / g_{i t}=5$ (row 1 ).

From this figure, it can be seen that, for a fixed ratio of $g_{i l} / g_{i t}$, increasing 
the ratio $g_{e t} / g_{i t}$ induces only slight changes in the epicardial potential distribution. For example, across the first row, there is only one peak in each potential distribution for most values of $g_{e t} / g_{i t}$ with three peaks appearing for $g_{e t} / g_{i t}=13$. Also, the distributions appear to become elongated in the $y$-direction and smaller in magnitude as this ratio increases. On the other hand, moving down the columns shows some significant changes. In the second row, the potential distributions are again similar to each other, with decreasing magnitude as the ratio increases. Across this row there are three peaks in each distribution, with the central peak lower than the outer two. In the third row, each distribution shows two peaks with column 2 having a third small peak in the centre, column three having two small valleys of negative potential which merge together towards the centre of the slab as the ratio $g_{e t} / g_{i t}$ increases (column 4). Finally, in the fourth row, negative valleys appear in each column and merge across the centre of the ischaemic region moving from left to right across the row. In summary, there are more significant changes moving down a column than there are moving across a row. It should also be pointed out that in this figure the potentials in the upper left hand data set are much higher than those from any other data set.

To further strengthen the observations from Figure 3, consider the correlation coefficients also displayed in the figure. It can be seen that while moving across a row, the correlation coefficients, calculated relative to column 2 (left hand value), do not reduce markedly. However, they do drop more rapidly moving across the row, as the ratio $g_{i l} / g_{i t}$ increases. There is a significant drop in the value of the correlation coefficients, calculated relative to row 1 (right hand value), observed while moving down a column. The drop in right hand correlation coefficients is much larger, moving down a column, than the decreases in left hand correlation coefficients, when moving across 
a row of Figure 3.

Figure 4 shows epicardial potential distributions, as described in Figure 3 , for the ratio $g_{b} / g_{i t}=15$. Similar comments to those regarding Figure 3 also apply here: moving across a row only induces small changes in the potential distributions, whereas moving down a column induces major changes. However, the main difference here is that the changes are not as significant as those in Figure 3. This is borne out by the correlation coefficients, which are much higher than the corresponding values in Figure 3.

Figures 5 and 6 show the epicardial potential distributions with the ratios $g_{b} / g_{i t}=25$ and $g_{b} / g_{i t}=35$, respectively. As was observed in the previous figures, increasing the ratio $g_{e t} / g_{i t}$, for a fixed ratio $g_{i l} / g_{i t}$, has a less dramatic effect than increasing the ratio $g_{i l} / g_{i t}$, for a fixed ratio $g_{e t} / g_{i t}$. The changes in Figure 5 are less dramatic than when $g_{b} / g_{i t}=10$ or 15 , but are more dramatic than when $g_{b} / g_{i t}=35$ (Figure 6 ). It can also be seen that the correlation coefficients of Figures 5 and 6 reduce less rapidly than those given in Figures 3 and 4 . Note that the lower left panel (indicated by *) of Figure 5 represents an approximation to the conductivity data of Roberts et al. [8] and the plot in column four, row 3 (indicated by ${ }^{*}$ ) in Figure 6 closely approximates the data of Clerc [7].

The potential distributions shown in the upper left plot of Figures 3, 4, 5 and 6 have potentials which are all significantly higher than those in any other distributions shown. The conductivity sets, giving rise to these plots, are all based on the data of Roberts and Scher.

The underlying change in moving from Figure 3 to Figure 6 is that the ratio $g_{b} / g_{i t}$ increases monotonically from 10 to 35 . These figures show that the effects of changing the ratios $g_{e t} / g_{i t}$ and $g_{i l} / g_{i t}$ are diminished as $g_{b} / g_{i t}$ increases. This also follows from a comparison of the correlation coefficients. 
That is, increasing the ratio $g_{b} / g_{i t}$ leads to less dramatic changes in the potential distributions.

\section{Discussion}

The results from the previous section show that, for a given ratio of $g_{b} / g_{i t}$, fixing the ratio $g_{i l} / g_{i t}$ and varying the ratio $g_{e t} / g_{i t}$ leads to reasonably small changes in epicardial potential distributions computed for a slab of cardiac tissue. Also, changes become smaller as the ratio $g_{b} / g_{i t}$ is increased.

On the other hand, for a given ratio of $g_{b} / g_{i t}$, fixing the ratio $g_{e t} / g_{i t}$ and varying the ratio $g_{i l} / g_{i t}$ leads to much more significant changes in the potential distributions. Again, these changes become less apparent as the ratio $g_{b} / g_{i t}$ is increased.

It could therefore be concluded that the ratio $g_{i l} / g_{i t}$ has a much more significant effect on the epicardial potential distribution than the ratio $g_{e t} / g_{i t}$. In other words, the intracellular conductivity along the fibre is much more important than the extracellular conductivity across the fibres in determining the resulting epicardial potential distribution.

These observations go some way to explaining the differences between the epicardial potential distributions obtained from the data sets of Clerc [7], Roberts et al. [8] and Roberts and Scher [9], since the first row of each of the figures presented (ignoring changes in the ratios $g_{e t} / g_{i t}$ and $g_{b} / g_{i t}$ ) is representative of the data of Roberts and Scher, whereas the entries in the third and fourth rows are representative of the data of Clerc and Roberts et al.. This is based on the fact that it does not appear to matter that these distributions are in different columns within the figures.

Varying the ratio $g_{e t} / g_{i t}$, for fixed $g_{b} / g_{i t}$ and $g_{i l} / g_{i t}$, has interesting consequences. The quantities $g_{e t}$ and $g_{b}$ are linked through the continuity of 
current boundary condition at the tissue-blood interface. It can be seen from equation (22) that increasing $g_{e t} / g_{i t}$ results in an increase in $g_{e l} / g_{i t}$, which effectively allows more current to travel through the entire extracellular space in the tissue in preference to passing into the blood. This explains the similarities in potential distributions as the ratio $g_{e t} / g_{i t}$ is increased.

Alternatively, increasing the ratio $g_{b} / g_{i t}$, for fixed $g_{e t} / g_{i t}$ and $g_{i l} / g_{i t}$, allows current to move more easily through the blood (i.e., the blood becomes more of a current sink at the tissue-blood interface). This has the effect of damping out the changes in epicardial potential distributions seen in moving through Figures $3,4,5$ and 6 , where the ratio $g_{b} / g_{i t}$ takes the values $10,15,25$ and 35 , respectively.

One way to overcome the shunting of current, through either the blood or the extracellular space, is to keep the ratio $g_{b} / g_{e t}$ reasonably constant and compare the resulting epicardial potential distributions, while varying the ratio $g_{i l} / g_{i t}$. By examining the input parameters, it can be seen that the pairs $g_{b} / g_{i t}=10$ and $g_{e t} / g_{i t}=5$, and $g_{b} / g_{i t}=25$ and $g_{e t} / g_{i t}=13$, both give a value for the ratio $g_{b} / g_{e t}$ of approximately 2. Further, the pairs $g_{b} / g_{i t}=15$ and $g_{e t} / g_{i t}=5$, and $g_{b} / g_{i t}=35$ and $g_{e t} / g_{i t}=13$, give a value of the ratio $g_{b} / g_{e t}$ of approximately 3. The potential distributions and correlation coefficients, obtained by comparing these distributions for various values of the ratio $g_{i l} / g_{i t}$, are given in Figure 7 . The columns for this figure comprise, from left to right, column 2 of Figure 3, column 4 of Figure 5, column 2 of Figure 4 and column 4 of Figure 6.

Since all the correlation coefficients in Figure 7 are very close to 1 , it can be concluded that even when the current continuity boundary condition is artificially adjusted to maintain a constant ratio of conductivity between the blood and the extracellular tissue, the most significant contribution to 
changes in the shape of the epicardial potential distributions is the ratio $g_{i l} / g_{i t}$. It should be noted that the magnitudes of the distributions do depend on the ratios $g_{b} / g_{i t}$ and $g_{e t} / g_{i t}$.

As mentioned previously [12], similarities in the ratio $g_{i l} / g_{i t}$ were used as a basis for explaining the similarities between the respective epicardial potential distributions based on the data sets of Clerc and Roberts et al. There are distinct differences between these distributions and that from the data of Roberts and Scher, as shown in Figure 2. The basis for this argument comes from the work of Hopenfeld et al. [5, 6], which states that the epicardial potential distribution depends on the ratio of the current flows across the lateral and transmural boundaries between normal and ischaemic regions of the tissue. Given the simple geometry studied here, it is straightforward to show that this ratio of currents is equivalent to the ratio $g_{i l} / g_{i t}[12]$.

In the context of the experimentally determined conductivity values, the data of Clerc [7] and Roberts et al. [8] give rise to tripolar epicardial potential distributions, with large potential gradients near the lateral ischaemic boundary, whereas those of Roberts and Scher generally yield a monopolar pattern without large potential gradients. This can be explained by the fact that, for Roberts and Scher, the value of $g_{i l} / g_{i t}$ is 5.7 , whereas the ratios are 9.0 and 10.8, respectively, for the other two data sets (see Table 1). Hence, for the Roberts and Scher data, there is reduced current flow across the lateral boundary of the ischaemic region compared to that over the transmural boundary. If the current flow across the lateral boundary could be increased, by increasing the degree of ischaemia, say [6], then it could be expected that a tripolar epicardial potential distribution and large potential gradients would result. This is indeed the case, as shown in Figure 8, which presents the epicardial potential distribution for the data of Roberts and Scher with $80 \%$ 
ischaemia.

\section{Conclusions}

A sensitivity study of cardiac tissue conductivity values has been undertaken to attempt to explain the differences between epicardial potential distributions observed when using various experimentally derived conductivity data sets $[7,8,9]$. The study was based on a recently presented nondimensional formulation of the passive bidomain equation [12] and considered a different parameter space to that considered previously [6].

It was found that varying the ratio $g_{e t} / g_{i t}$ for fixed $g_{i l} / g_{i t}$ and $g_{b} / g_{i t}$ gave rise to only slight changes in the epicardial potential distribution. This was observed via visual comparisons of the distributions presented and supported by calculations of the correlation coefficient. However, varying the ratio $g_{i l} / g_{i t}$ for fixed $g_{e t} / g_{i t}$ and $g_{b} / g_{i t}$, showed significant variation in the epicardial potential distributions, which movedg from one to three peaks in the distribution as $g_{i l} / g_{i t}$ increased. Further, fixing the ratio $g_{b} / g_{e t}$ showed that the epicardial potential distributions changed only with the ratio $g_{i l} / g_{i t}$.

It should also be stated that the other ratios studied do affect the epicardial potential distribution, but it appears that the most significant effects are produced by the ratio $g_{i l} / g_{i t}$.

This study shows that variations in the ratio $g_{i l} / g_{i t}$ are most likely to explain the differences in epicardial potential distributions obtained from the three experimentally determined conductivity data sets. The data of Clerc yield $g_{i l} / g_{i t}=9.0$ and the data of Roberts et al. yield $g_{i l} / g_{i t}=10.8$ and both of these give three peaks in the epicardial potential distribution. The data of Roberts and Scher give $g_{i l} / g_{i t}=5.7$, which induces only one peak in the epicardial potential distribution. 
The data of Roberts and Scher are at the corner of the parameter space considered here, whereas the other two data sets lie on "faces" of the three dimensional parameter space. From a biophysics point of view, Roberts and Scher's data induce a much lower ratio of lateral to transmural boundary current flow from normal to ischaemic region than the other data sets. Further, this data set fails to give the large potential gradients which are observed experimentally above the borders of the ischaemic regions [16]. Given the extreme nature of this data set, perhaps it is appropriate to consider these conductivity values as outliers.

There have also been questions raised over the values suggested by Clerc $[11,24]$. According to [11], Clerc's reported value for $g_{e l}$ would imply nonphysiologically high concentrations of sodium or chloride ions in the interstitial fluid. Further, in [24], the model underlying the analysis of Clerc's experimental data is considered to be inadequate.

In light of the above discussion, it is suggested that it might be most appropriate to use the data of Roberts et al. [8] for future simulation studies. This is consistent with recent work [12], based on simple modelling, which suggested taking $g_{i l} / g_{i t}=10$ and $g_{e t} / g_{i t}=5.25$, values that are very close to the ratios obtained from the data of Roberts et al..

Conflict of Interests: None 


\section{References}

[1] P. R. Johnston, D. Kilpatrick, C. Y. Li, The importance of anisotropy in modelling ST segment shift in subendocardial ischaemia, IEEE Transactions on Biomedical Engineering 48 (12) (2001) 1366-1376.

[2] P. R. Johnston, Tissue conductivity and ST depression in a cylindrical left ventricle, International Journal of Bioelectromagnetism 4 (2) (2002) $61-62$

[3] P. R. Johnston, D. Kilpatrick, The effect of conductivity values on ST segment shift in subendocardial ischaemia, IEEE Transactions on Biomedical Engineering 50 (2) (2003) 150-158.

[4] P. R. Johnston, A cylindrical model for studying subendocardial ischaemia in the left ventricle, Mathematical Biosciences 186 (1) (2003) $43-61$.

[5] B. Hopenfeld, J. G. Stinstra, R. S. MacLeod, Mechanism for ST depression associated with contiguous subendocardial ischaemia, Journal of Cardiovascular Electrophysiology 15 (2004) 1200-1206.

[6] B. Hopenfeld, J. G. Stinstra, R. S. MacLeod, The effect of conductivity on st-segment epicardial potentials arising from subendocardial ischemia, Annals of Biomedical Engineering 33 (6) (2005) 751-763.

URL http://dx.doi.org/10.1007/s10439-005-3236-2

[7] L. Clerc, Directional differences of impulse spread in trabecular muscle from mammalian heart, Journal of Physiology 255 (1976) 335-346.

[8] D. E. Roberts, L. T. Hersh, A. M. Scher, Influence of cardiac fiber ori- 
entation on wavefront voltage, conduction velocity and tissue resistivity in the dog, Circ. Res. 44 (1979) 701-712.

[9] D. E. Roberts, A. M. Scher, Effects of tissue anisotropy on extracellular potential fields in canine myocardium in situ, Circ. Res. 50 (1982) 342351.

[10] J. G. Stinstra, B. Hopenfeld, R. MacLeod, A model for the passive cardiac conductivity, International Journal of Biolectromagnetism 5 (1) (2003) 185-186.

[11] J. Stinstra, B. Hopenfeld, R. MacLeod, On the passive cardiac conductivity, Annals of Biomedical Engineering 33 (12) (2005) 1743-1751.

URL http://www.springerlink.com/openurl. asp?genre= article\&id=doi:10.1007/s10439-005-7257-7

[12] P. R. Johnston, A non-dimensional formulation of the passive bidomain equation, Journal of Electrocardiology 44 (2) (2011) 184-188.

[13] R. M. Gulrajani, Bioelectricity and Biomagnetism, John Wiley and Sons, New York, 1998.

[14] M. Potse, B. Dubé, A. Vinet, Cardiac anisotropy in boundary-element models for the electrocardiogram, Medical \& Biological Engineering \& Computing 47 (7) (2009) 719-729.

URL http://www.springerlink.com.libraryproxy.griffith.edu. $\mathrm{au} /$ content/y677n572wp1353h0/

[15] L. Tung, A bi-domain model for describing ischaemic myocardial D-C potentials, Ph.D. thesis, Massachusetts Institute of Technology (June 1978). 
[16] D. Li, C. Y. Li, A. C. Yong, D. Kilpatrick, Source of electrocardiographic ST changes in subendocardial ischemia, Circulation Research 82 (1998) 957-970.

[17] B. J. Roth, A comparison of two boundary conditions used with the bidomain model of cardiac tissue, Annals of Biomedical Engineering 19 (1991) 669-678.

[18] S. G. Patel, B. J. Roth, Approximate solution to the bidomain equations for electrocardiogram problems, Physical Review E (Statistical, Nonlinear, and Soft Matter Physics) 72 (5) (2005) 051931.

URL http://link.aps.org/abstract/PRE/v72/e051931

[19] P. R. Johnston, Approximate solutions for certain bidomain problems in electrocardiography, Physical Review E 78 (4). doi:http://dx.doi.org/10.1103/PhysRevE.78.041904.

URL http://link.aps.org/abstract/PRE/v78/e041904

[20] S. Rush, J. A. Abildskov, R. McFee, Resistivity of body tissues at low frequencies, Circulation Research 12 (1963) 40-50.

[21] P. R. Johnston, A finite volume method solution for the bidomain equations and their application to modelling cardiac ischaemia, Computer Methods in Biomechanics and Biomedical Engineering 13 (2) (2010) 157-170. doi:10.1080/10255840903067072.

[22] D. Kilpatrick, A. J. Bell, S. J. Walker, Derived epicardial potentials differentiate ischemic ST depression from ST depression secondary to ST elevation in acute inferior myocardial infarction in man, Journal of the American College of Cardiology 14 (1989) 695-702. 
[23] D. D. Streeter, Gross morphology and fiber geometry of the heart, in: R. M. Berne (Ed.), Handbook of Physiology, Vol 1, Williams and Williams, Baltimore, MD, 1979, Ch. 2: The Cardiovascular System, pp. $61-112$.

[24] R. Plonsey, A. van Oosterom, Implications of macroscopic source strength on cardiac cellular activation models, Journal of Electrocardiology 24 (2) (1991) 99-112. 


\section{Table and Figure Captions}

Table 1: Conductivity data for the three indicated experimental studies (given in $\mathrm{S} / \mathrm{m}$ ) and derived quantities used in this study.

Figure 1: A schematic view of the tissue-blood model used in this study. The left hand panel is a cross-section view of the tissue $(0 \leq z \leq 1)$ and the blood $(z>1)$ with the positive $z$ axis pointing downwards. The hashed region indicates ischaemic tissue. The right hand panel is a plan view of the model, looking from the $-z$ direction, again with the hashed region indicating the ischaemic tissue. The dashed arrow indicates the fibre direction on the endocardium (at $z=1$ ) when the fibre direction on the epicardium (at $z=0$ ) points along the $x$-axis.

Figure 2: Dimensionless epicardial potential distributions in a slab model of cardiac tissue for three different conductivity data sets. Panel (a) is generated from the data of Clerc [7], panel (b) is generated from the data of Roberts et al. [8] and panel (c) is generated from the data of Roberts and Scher [9]. Positive potentials are indicated by thin solid lines with the zero of potential represented by a thick solid line.

Figure 3: Dimensionless epicardial potential distributions in a slab model of cardiac tissue for different conductivity ratios $g_{i l} / g_{i t}$ and $g_{e t} / g_{i t}$ for a fixed ratio of $g_{b} / g_{i t}=10$. The columns correspond to values of the ratio $g_{e t} / g_{i t}$ of 1, 5, 9 and 13, respectively, and the rows correspond to values of the ratio $g_{i l} / g_{i t}$ of $5,7,9$ and 11 , respectively. Positive potentials are indicated by solid lines and negative potentials are indicated by dashed lines, with the thick solid line indicating the zero of potential. The contour interval is 0.005 , except in the upper left plot where it is 0.05 . The numbers below each figure indicate correlation coefficients as described in the text. The panel indicated by the ${ }^{*}$ is an approximation to the data of Roberts and Scher [9]. 
Figure 4: Dimensionless epicardial potential distributions in a slab model of cardiac tissue for different conductivity ratios $g_{i l} / g_{i t}$ and $g_{e t} / g_{i t}$ for a fixed ratio of $g_{b} / g_{i t}=15$. The format is the same as for Figure 3 .

Figure 5: Dimensionless epicardial potential distributions in a slab model of cardiac tissue for different conductivity ratios $g_{i l} / g_{i t}$ and $g_{e t} / g_{i t}$ for a fixed ratio of $g_{b} / g_{i t}=25$. The format is the same as for Figure 3. The panel indicated by the ${ }^{*}$ is an approximation to the data of Roberts et al. [8].

Figure 6: Dimensionless epicardial potential distributions in a slab model of cardiac tissue for different conductivity ratios $g_{i l} / g_{i t}$ and $g_{e t} / g_{i t}$ for a fixed ratio of $g_{b} / g_{i t}=35$. The format is the same as for Figure 3. The panel indicated by the $*$ is an approximation to the data of Clerc [7].

Figure 7: Dimensionless epicardial potential distributions in a slab model of cardiac tissue for different conductivity ratios $g_{i l} / g_{i t}$ for two different values of the ratio $g_{b} / g_{e t}$. The two columns on the left are for $g_{b} / g_{e t} \approx 2$ and the two columns on the right are for $g_{b} / g_{e t} \approx 3$. The numbers under each pair of distributions give the correlation coefficients between the two distributions.

Figure 8: Dimensionless epicardial potential distributions in a slab model of cardiac tissue for the conductivity data of Roberts and Scher [9] and 80\% subendocardial ischaemia. 


\begin{tabular}{|c|c|c|c|}
\hline Quantity & $\begin{array}{c}\text { Clerc } \\
(1976)\end{array}$ & $\begin{array}{c}\text { Roberts } \text { et al. } \\
(1979)\end{array}$ & $\begin{array}{c}\text { Roberts \& Scher } \\
(1982)\end{array}$ \\
\hline$g_{i l}$ & 0.17 & 0.28 & 0.34 \\
$g_{i t}$ & 0.019 & 0.026 & 0.06 \\
$g_{e l}$ & 0.62 & 0.22 & 0.12 \\
$g_{e t}$ & 0.24 & 0.13 & 0.08 \\
\hline$\delta$ & 3.13 & 3.21 & 3.29 \\
\hline$g_{i l} / g_{i t}$ & 9.0 & 10.8 & 5.7 \\
$g_{e t} / g_{i t}$ & 12.3 & 5.0 & 1.3 \\
$g_{b} / g_{i t}$ & 32.3 & 23.8 & 10.5 \\
\hline
\end{tabular}

Table 1:

\begin{tabular}{c|cc}
\hline Tissue & & $z=0$ \\
& & \\
\hline Blood & & $z=1$ \\
& $z$ &
\end{tabular}

Figure 1: 


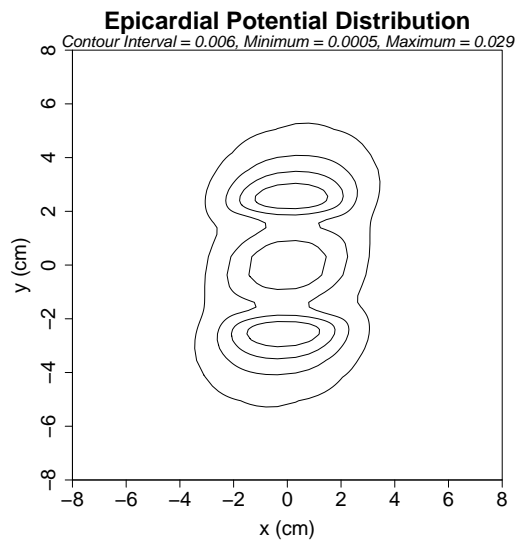

(a) Clerc (1976) [7]

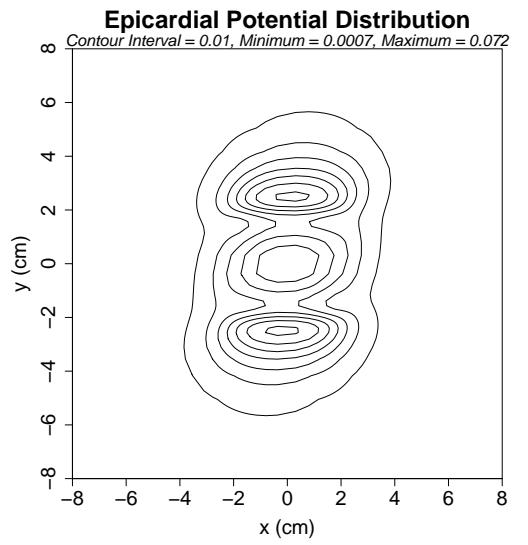

(b) Roberts et al. (1979) [8]

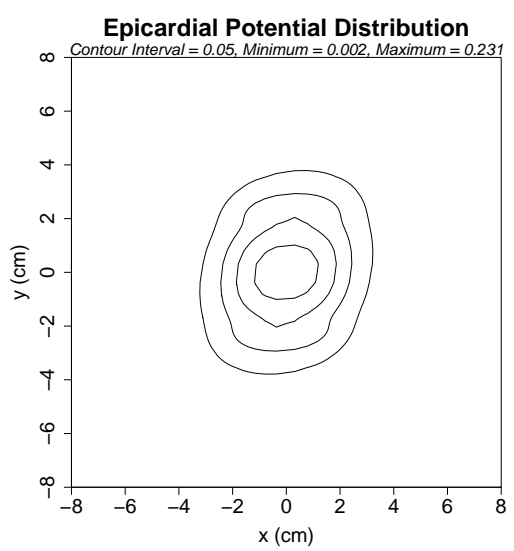

(c) Roberts \& Scher (1982) [9]

Figure 2: 


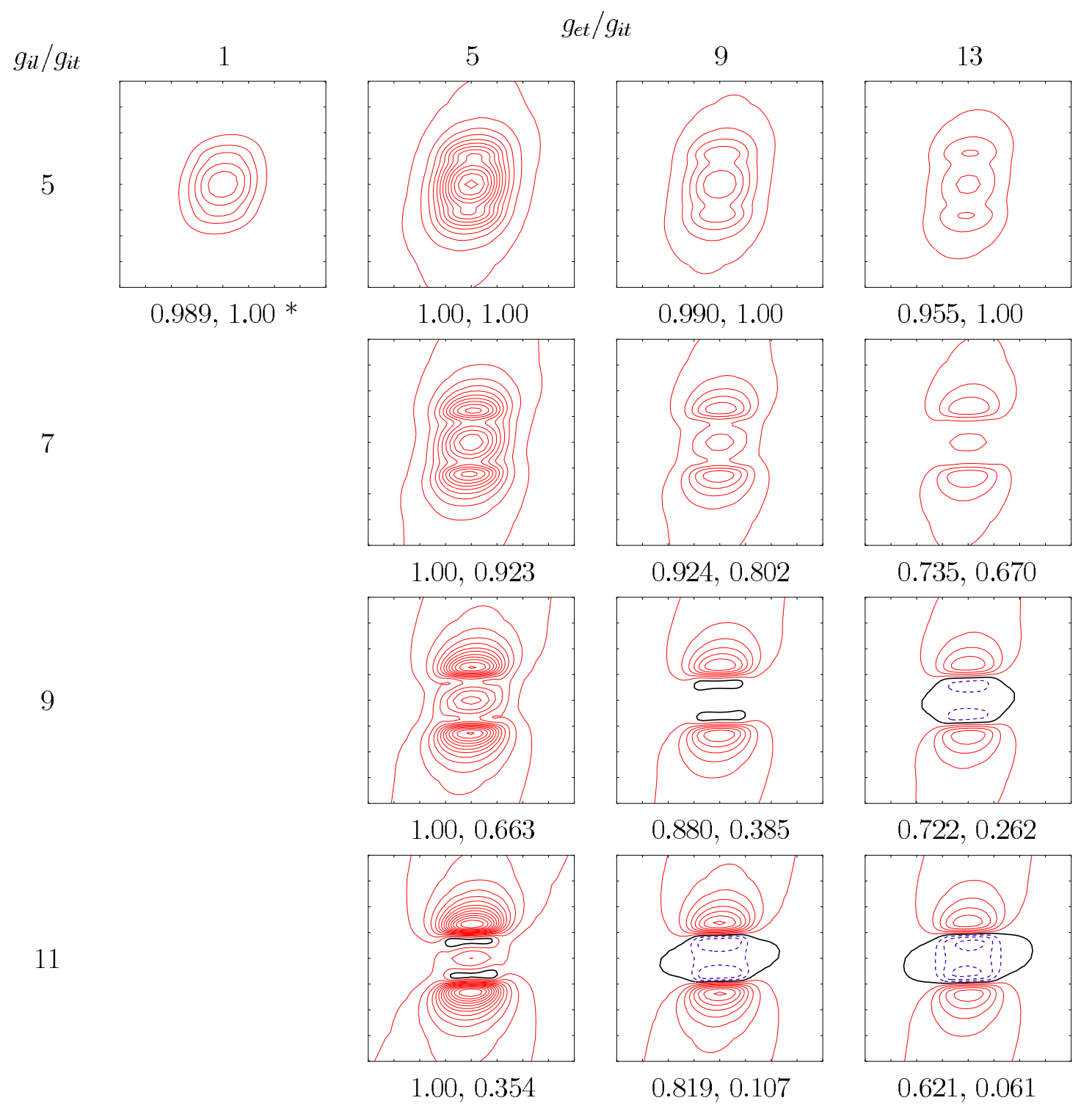

Figure 3: 


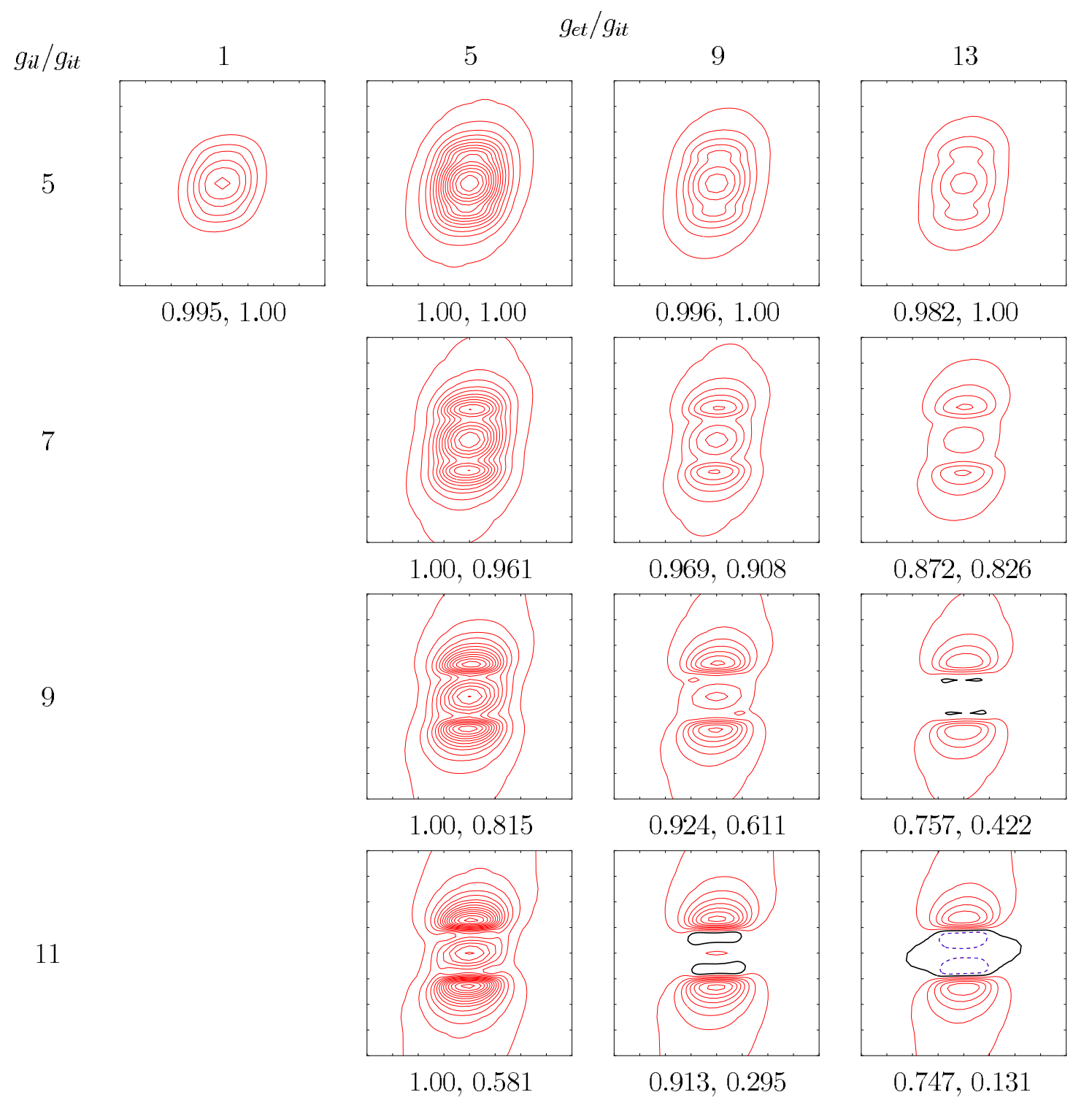

Figure 4: 


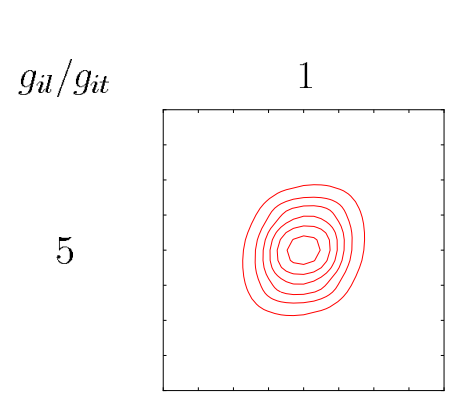

$0.998,1.00$

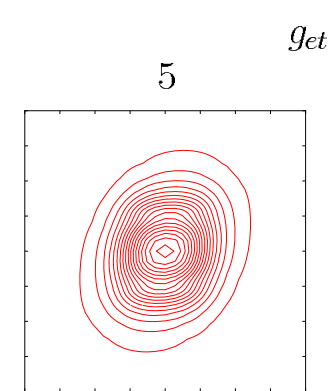

$1.00,1.00$

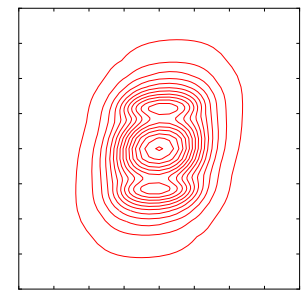

$1.00,0.980$

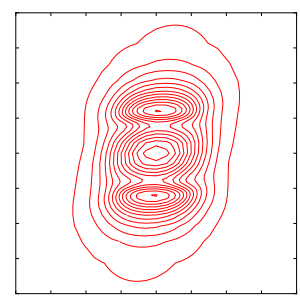

$1.00,0.905$

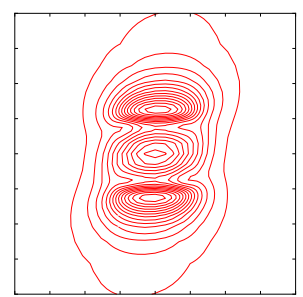

$1.00,0.766^{*}$ $g_{e t} / g_{i t}$

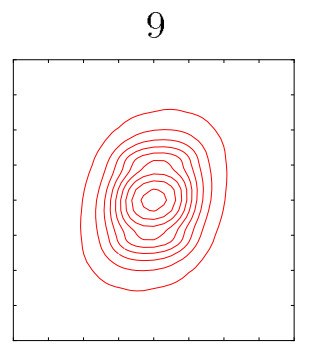

$0.998,1.00$

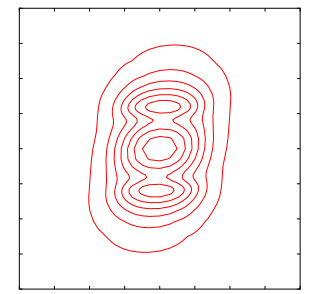

0.990, 0.961

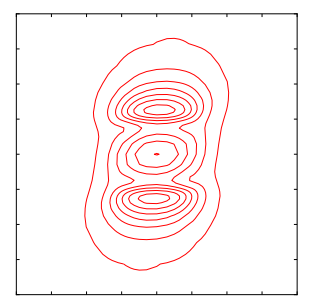

$0.974,0.815$

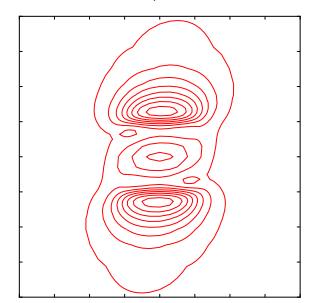

0.951, 0.582
13

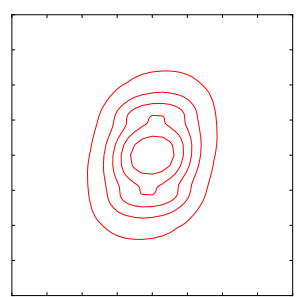

$0.993,1.00$

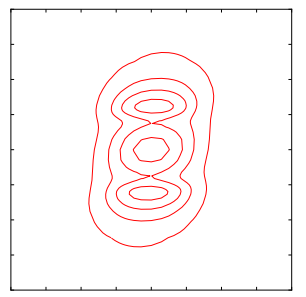

$0.960,0.933$

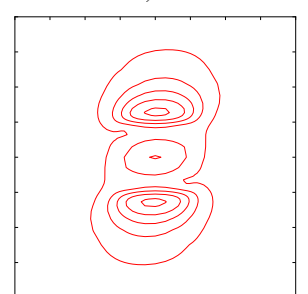

$0.888,0.697$

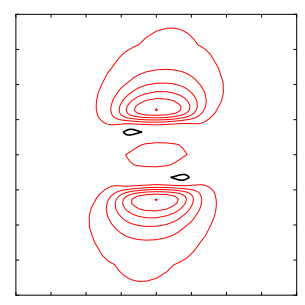

0.833, 0.398

Figure 5: 


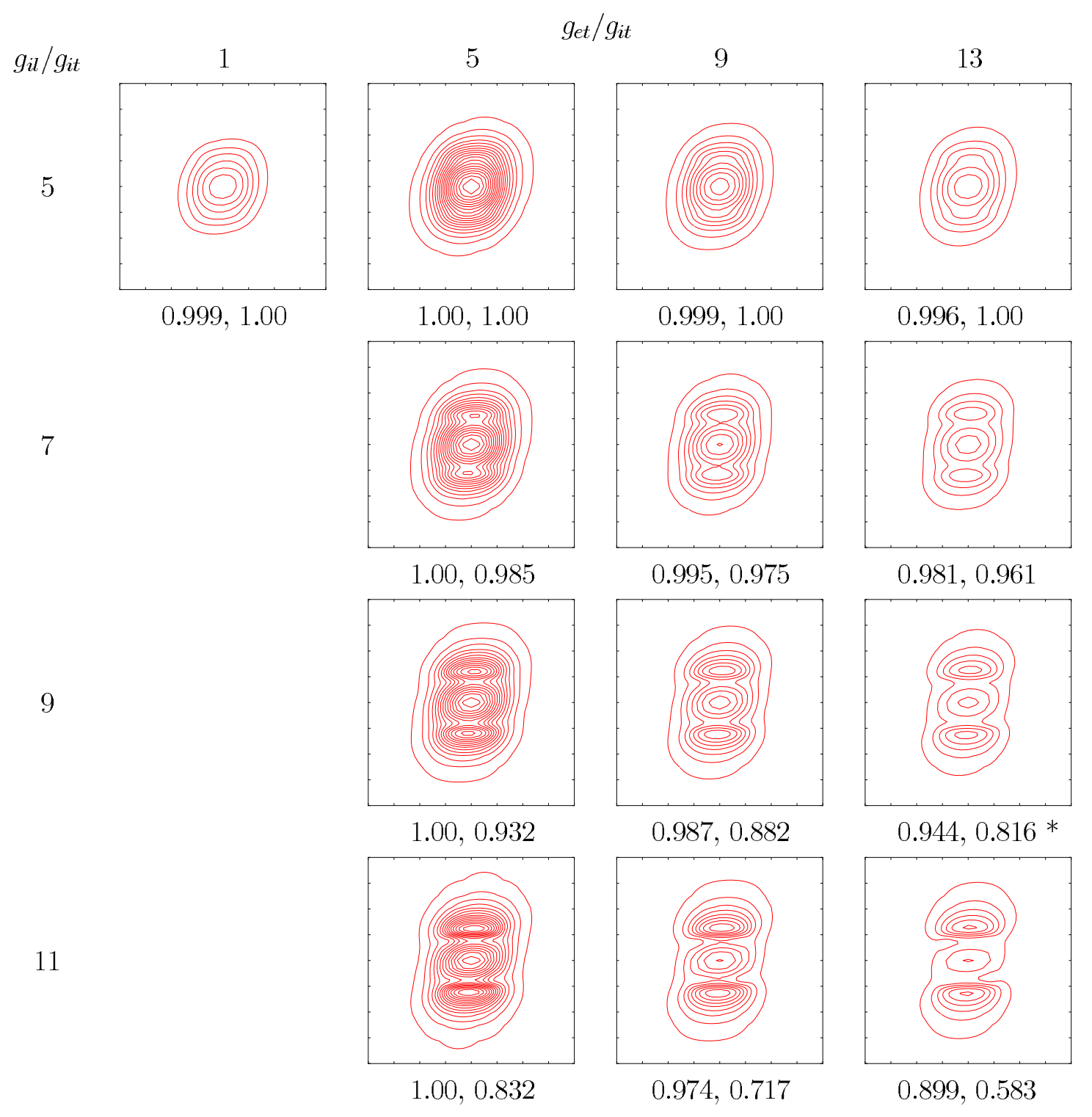

Figure 6: 


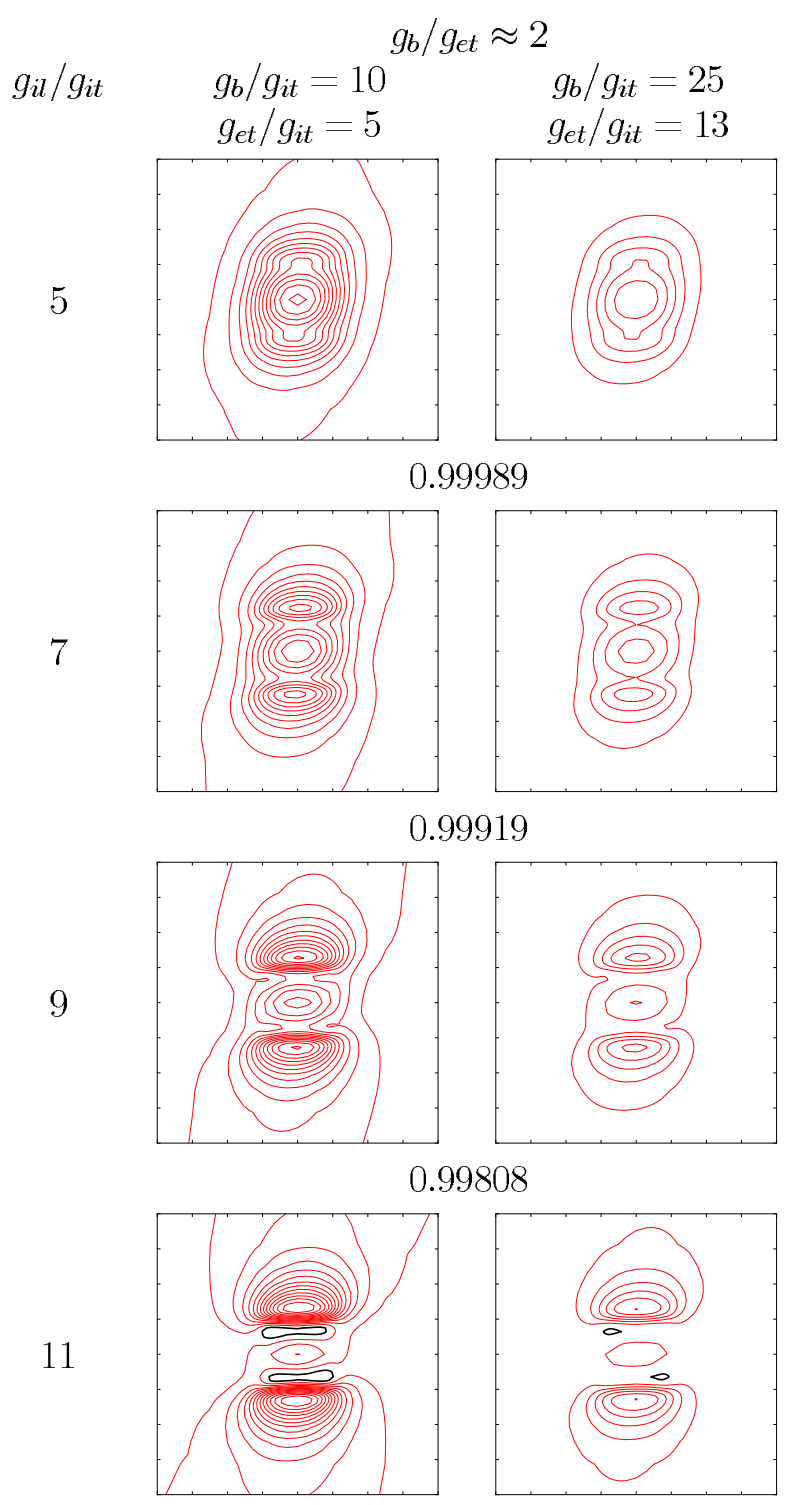

0.99803

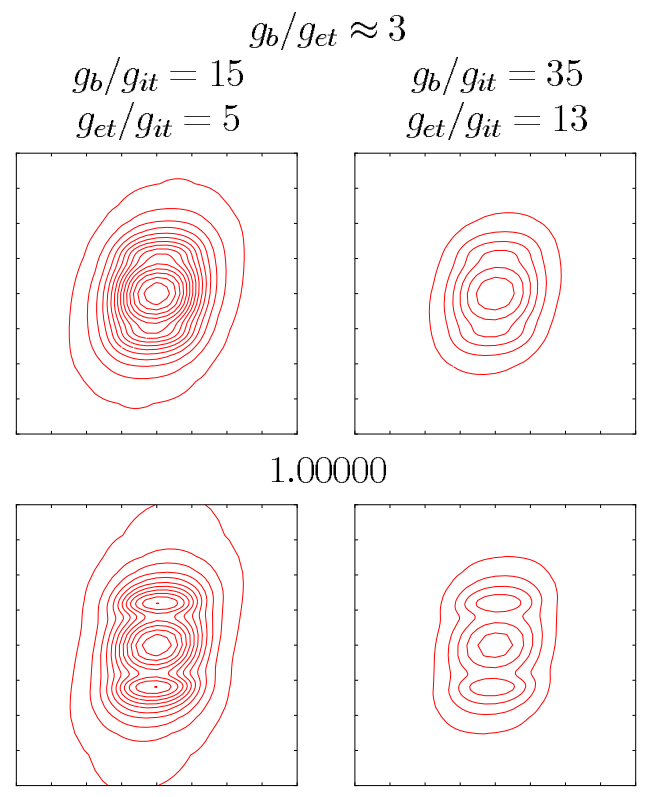

0.99999

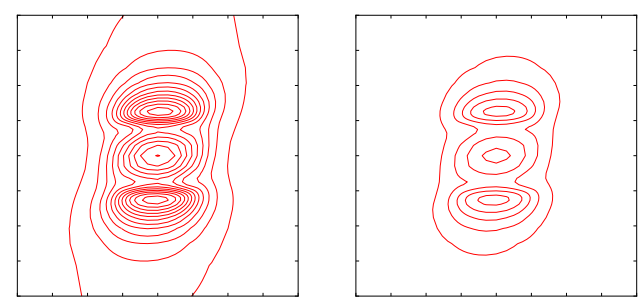

0.99997

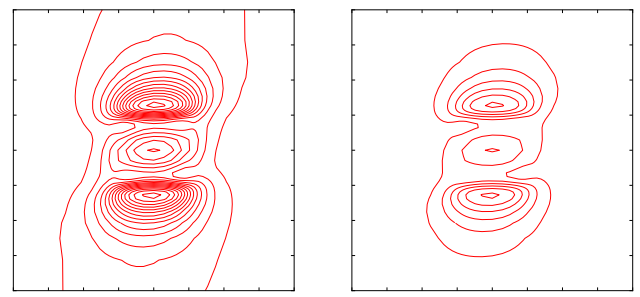

0.99994

Figure 7: 


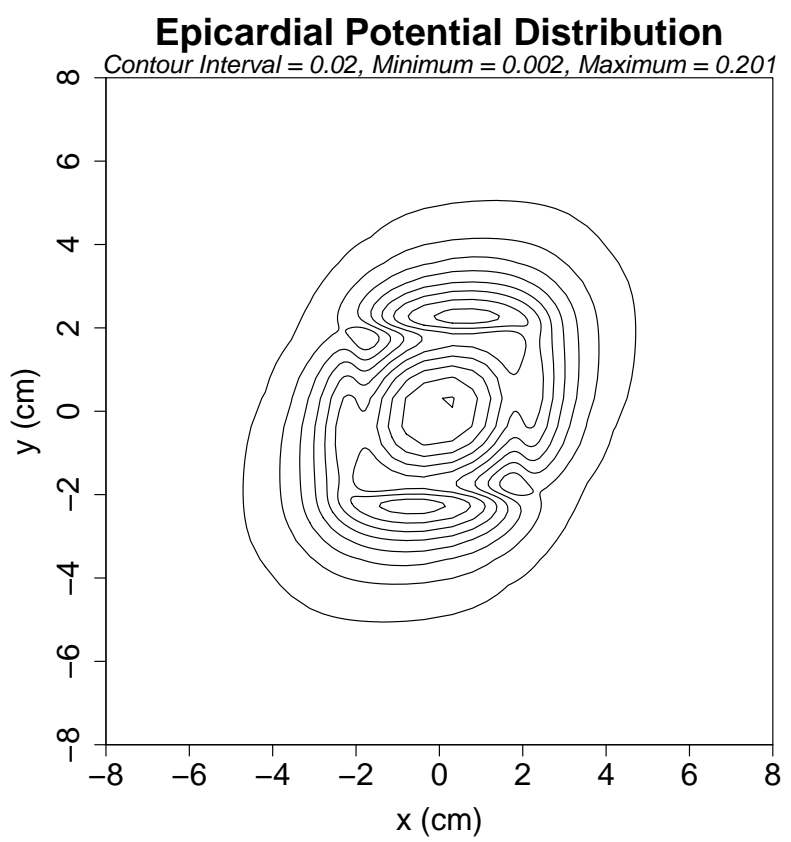

Figure 8: 